\title{
Analysis of Endocardial Micro-Accelerometry during Valsalva Maneuvers
}

\author{
Clément Gallet ${ }^{1,2}$, Virginie Le Rolle ${ }^{1,2}$, Jean-Luc Bonnet ${ }^{3}$, Christine Henry ${ }^{3}$, A. Hagège ${ }^{4}$, \\ Philippe Mabo ${ }^{1,2,5}$, Guy Carrault ${ }^{1,2}$, A.I. Hernández ${ }^{1,2}$ \\ ${ }^{1}$ INSERM, U1099, Rennes, F-35000, France. \\ ${ }^{2}$ Université de Rennes 1, LTSI, Rennes, F-35000, France. \\ ${ }^{3}$ Sorin CRM SAS (a LivaNova company), Clamart, France. \\ ${ }^{4}$ INSERM, UMR970 Paris Cardiovascular Research Center, Paris, France. \\ ${ }^{5}$ CHU Rennes, Department of Cardiology, Rennes, F- 35000, France.
}

\begin{abstract}
The endocardial acceleration (EA) signal is a particular kind of cardiac mechanogram, acquired through a microaccelerometer, embedded at the tip of a cardiac pacing lead, that can be chronically implanted inside a cardiac chamber. In this work, we analyze the evolution of the main EA-derived markers during severe cardio-respiratory modifications. Intracardiac electrograms (EGM), intraventricular pressure and EA data were acquired from 6 sheep during a set of Valsalva-like maneuvers. These data have been processed to segment the main EA components during each Valsalva phase, in a beat-to-beat basis. Results confirm the correlation between the energy of the EA1 component and the dP/dtmax. Significant modifications are observed in the main components of the EA signal during and just after the maneuver. These modifications may be useful for the characterization of respiratory events from the analysis of intracardiac EA signals.
\end{abstract}

\section{Introduction}

Cardiac mechanoacoustic signals have been shown to provide useful information for the evaluation of the mechanical function of the heart [1]. In particular, the Endocardial Acceleration (EA) signal, which can be acquired chronically from inside a cardiac chamber through a micro-accelerometer embedded at the tip of a pacing lead, has opened new possibilities for long-term monitoring of the cardiac electromechanical function of patients with an implantable device.

Preliminary experimental works have shown that the EA signal provides useful information about the inotropic state, showing that the peak-to-peak amplitude of the first EA component (EA1), which is synchronous with the first heart sound, is significantly correlated with the maximum derivative of the left ventricular pressure signal [2]. This amplitude has also been shown to decrease in humans during acute myocardial ischemia, where myocardial contractility is known to be decreased [3]. More recently, our group has proposed EA processing methods allowing for the estimation of cardiac electro-mechanical parameters, which have been applied to the improvement of patient selection and to optimize device configuration in the context of Cardiac Resynchronization Therapy (CRT) [4-7].

In this work, we analyze the evolution of the main EAderived markers during severe cardio-respiratory modifications, in order to identify robust variables that may be useful for long-term patient monitoring or for the optimization of closed-loop therapies [8]. Intracardiac electrograms (EGM), intra-ventricular pressure and EA data obtained from the right ventricle, were acquired from 6 sheep during a set of Valsalva-like maneuvers. These data have been processed offline to estimate the inotropic state and to segment the main EA components in a beat-to-beat basis. After a description of the experimental protocol, the signal processing methods for EGM, ventricular pressure and EA are briefly exposed. Results illustrate the variation of the extracted markers during the different phases of the Valsalva-like maneuver and the correlation between these different markers.

\section{Methods}

\subsection{Experimental protocol}

The experimental protocol in this study was approved by the French ethics committee for animal experimentation (Comité d'éthique en matiere d'expérimentation animale Paris Descartes, Paris, France). Experiments were performed on 6 sheep with a myocardial infarction (MI) provoked by ligatures on three marginal coronary arteries. The following protocol was applied two weeks after the induction of MI. Under isoflurane anesthesia (2\%) and con- 
trolled ventilation, a multi-sensor lead (Millar Instruments, Inc., Houston, USA) was inserted into the left ventricle for the acquisition of intracardiac pressure and EGM. A second lead (SonRTip, Sorin Group Italia, Saluggia, Italy) for measuring EA was inserted into the right ventricle. All signals were recorded and sampled at $1 \mathrm{kHz}$. After the placement of intracardiac leads, anesthesia was changed to etomidate $(100 \mu \mathrm{g} / \mathrm{kg} / \mathrm{min})$ in order to preserve the autonomic response during the rest of the experiment. During the whole procedure, animals were kept under artificial respiration, that was modified to provoke the Valsalva-like maneuvers.

\subsection{Valsalva-like maneuver}

Once cardiovascular parameters were stabilized, a Valsalva-like maneuver [9] was triggered three times for each sheep, separated by at least 60 seconds. Each maneuver consists in two steps.

CPP step: an apnea with a continuous positive pressure (CPP) of $30 \mathrm{cmH}_{2} \mathrm{O}$ was applied during $15 \mathrm{~s}$. The increased intrathoracic pressure during this step provokes a transient effect of increased blood pressure (phase I) because of the mechanical compression of the intrathoracic vessels and the aorta. Then, the lowered venous return causes a lowered cardiac preload and a decreased systolic pressure (phase II) which is then compensated by the arterial baroreceptor reflex [9]. Systolic pressure is then stabilized, reflex heart rate and peripheral resistance increase.

Apnea step: CPP is stopped and followed by an apnea at atmospheric pressure for $10 \mathrm{~s}$. The release of the intrathoracic strain briefly causes a further fall in blood pressure, because pulmonary vessels are filled again (phase III). Then, venous return causes an increase in cardiac output. Together with the high peripheral resistance, this results in a pressure overshoot, which triggers a reflex bradycardia (phase IV). Finally, normal conditions are restored.

\subsection{Data processing}

R-waves were detected off-line from the EGM using a custom algorithm, with parameters adapted to the sheep [10]. RR intervals were visually examined and arrhythmic events were removed. Systolic and diastolic pressures were also automatically extracted from the left ventricular pressure signal. The rate $\mathrm{dP} / \mathrm{dt}_{\max }$ was then computed as the maximum derivative of the left ventricular pressure signal, on a beat-to-beat basis. This index reflects the contractile function of the heart and is recognized as a robust marker of inotropism [11]. The rate $\mathrm{dP} / \mathrm{dt}_{\min }$ was also computed, which depends on the relaxation function and the peak systolic left ventricular stress [12].

Four components can be identified during a single beat on the EA signal, corresponding to the four cardiac sounds on phonocardiography [2]: the closure of atrioventricular valves during the beginning of the systole (EA1), the closure of aortic and pulmonary valves (EA2), the end of the rapid filling of the ventricles (EA3) and the ventricular filling following the atrial contraction (EA4). In the sheep, the two last components were not distinguishable, and thus were grouped as component EA3/4. The EA signal was decomposed into beat segments using the previously detected $\mathrm{R}$ waves as reference. Then, 15 consecutive beat segments were aligned by maximizing their correlation, and averaged. The obtained average cycles were processed as described in $[6,7]$, so as to obtain the time instant of the beginning of each component $\left(t_{E A 1}, t_{E A 2}\right.$ and $\left.t_{E A 3 / 4}\right)$, the energy of the signal during each EA component $\left(E_{E A 1}\right.$, $E_{E A 2}$ and $\left.E_{E A 3 / 4}\right)$ and the width of the EA1 and EA2 components $\left(w_{E A 1}, w_{E A 2}\right)$.

All markers derived from the three recorded signals were averaged for each Valsalva maneuver during a 25-s baseline period before the onset of the maneuver and during the two phases of the maneuvers. Statistical comparisons between the baseline and each Valsalva step, as well as between the CPP and apnea steps were computed using a paired Wilcoxon signed rank test. P-values lower than 0.05 were considered statistically significant.

\section{Results}

The three available Valsalva-like maneuvers from each one of the 6 sheep were processed, leading to a total of $\mathrm{n}=18$ data segments to be analyzed. A representative example of an averaged beat cycle, showing the three recorded signals, is depicted in Figure 1. The main EA components analyzed in this study are labelled in the lower panel. The following sections present the analysis of how parameters extracted from these averaged beats evolve through the applied autonomic maneuver.
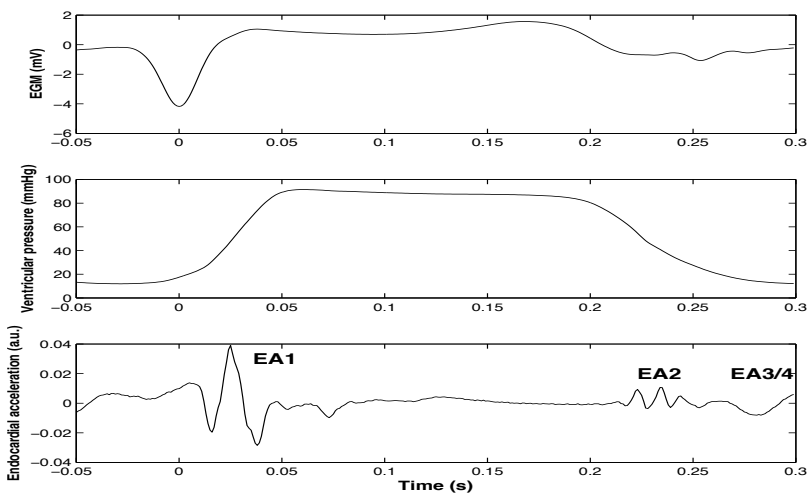

Figure 1. Beat-aligned and averaged signals of EGM, left ventricular pressure and endocardial acceleration (EA) obtained during a continuous baseline segment of $25 \mathrm{~s}$. The three components of the EA signals are shown. 

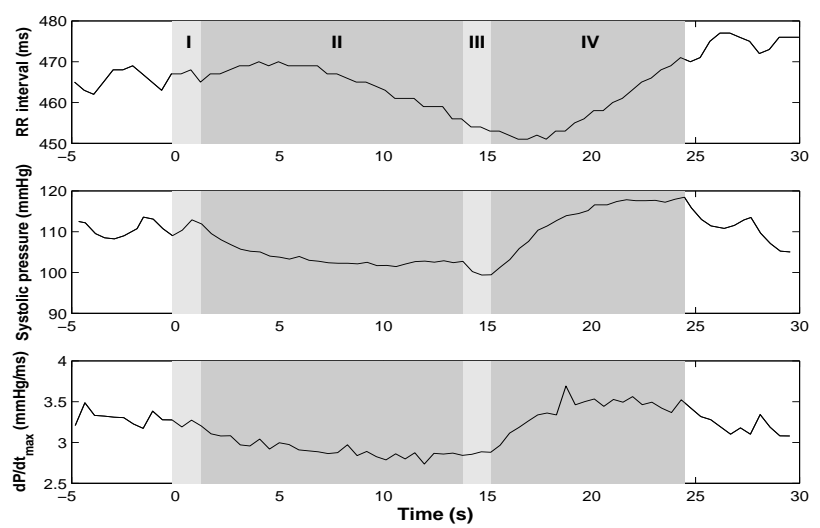

Figure 2. Time series representing the evolution of RR intervals, systolic pressure and $\mathrm{dP} / \mathrm{dt}_{\max }$ during a single Valsalva maneuver in one sheep. The four phases of the Valsalva maneuver are indicated.

Figure 2 shows a representative Valsalva-like recording. The main phases of the maneuver are depicted and the characteristic evolution of the main cardiovascular parameters can be observed in this example. Mean values of the main cardiovascular variables during significant phases of the Valsalva maneuvers are shown in Table 1. As expected, all cardiovascular variables presented significant modifications. The systolic pressure followed an exponential decrease during CPP, and was stabilized at the end of phase II. The $\mathrm{dP} / \mathrm{dt}_{\max }$ index was significantly lowered during CPP (phase II) and increased during apnea (phase IV) with respect to the baseline level. Similar results were observed for $\mathrm{dP} / \mathrm{dt}_{\text {min }}$, but with the opposite sign.

Table 1. Mean values of cardiovascular variables during baseline and Valsalva phases (mean $\pm \mathrm{SD}, \mathrm{n}=18$ ). $P_{S}=$ systolic pressure. $P_{D}=$ diastolic pressure. $* p<0.05$ vs baseline; $\dagger p<0.05$ vs CPP.

\begin{tabular}{|c|c|c|c|}
\hline & Baseline & CPP & Apnea \\
\hline $\mathrm{RR}(\mathrm{ms})$ & $472 \pm 38$ & $462 \pm 32^{*}$ & $451 \pm 33^{* \dagger}$ \\
\hline$P_{S}(\mathrm{mmHg})$ & $157 \pm 78.1$ & $138 \pm 72^{*}$ & $159 \pm 81^{\dagger}$ \\
\hline$P_{D}(\mathrm{mmHg})$ & $11 \pm 8$ & $15 \pm 10^{*}$ & $10 \pm 8^{* \dagger}$ \\
\hline $\mathrm{dP} / \mathrm{dt}_{\max }, \mathrm{mmHg} / \mathrm{ms}$ & $3.8 \pm 1.8$ & $3.4 \pm 2.1^{*}$ & $4.2 \pm 2.0^{* \dagger}$ \\
\hline $\mathrm{dP} / \mathrm{dt}_{\min }, \mathrm{mmHg} / \mathrm{ms}$ & $-2.6 \pm 1.7$ & $-1.9 \pm 1.3^{*}$ & $-2.5 \pm 1.7^{* \dagger}$ \\
\hline
\end{tabular}

The EA signal showed noticeable morphological variations during Valsalva maneuvers, as shown on one sheep in Figure 3. Note the amplitude modifications of all EA components during CPP and apnea and, in particular, the marked increase during apnea with respect to CPP.

Figure 4 shows the evolution of the main EA markers, for the three repetitions of the Valsalva-like maneuver and for one representative sheep. This evolution was reproducible for the three realizations of the maneuver on each sheep. The intra-experiment variability, computed as the

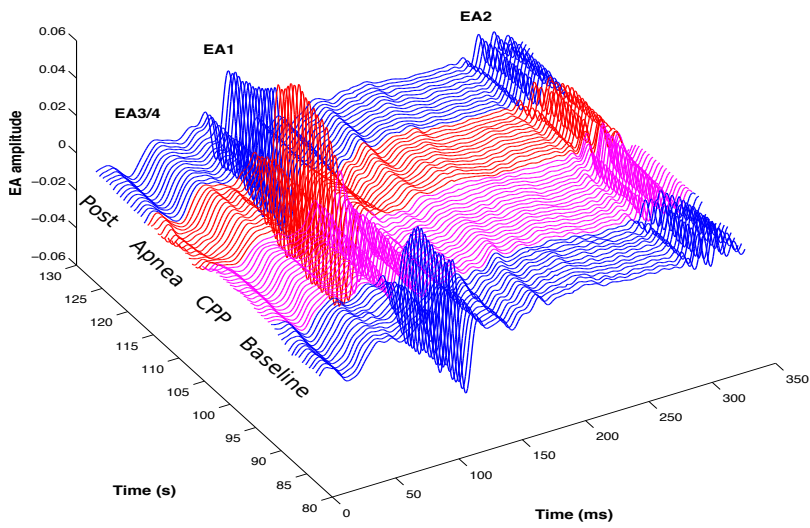

Figure 3. Modifications of the EA signal morphology (averaged cycles) during a single Valsalva maneuver.

mean variance of EAx energy between maneuvers, was $4.0 \times 10^{-3}, 2.6 \times 10^{-5}$ and $1.5 \times 10^{-6}$, respectively for $E_{E A 1}, E_{E A 2}$ and $E_{E A 3 / 4}$. These are much lower than the inter-experiment variability $\left(3.7 \times 10^{-2}, 8.5 \times 10^{-4}\right.$ and $\left.1.2 \times 10^{-4}\right)$.

Mean values of the main EA markers calculated for all sheep are shown in Table 2, along with results from the paired Wilcoxon test. Note that data on Table 2 represents the global mean value of each marker and this may hide the differences observed in a paired analysis. $t_{E A 1}$ and $t_{E A 2}$ were shortened during CPP and apnea, compared to baseline, which is consistent with the known evolution of the pre-ejective period during these phases. Significant EA energy increases were observed between baseline and apnea for all EA components. $E_{E A 1}$ and $E_{E A 2}$ were also statistically different between CPP and apnea. Only $E_{E A 3 / 4}$ showed a significant decrease between the baseline and CPP steps (results for $E_{E A 1}$ were close to the significance threshold). These modifications may be related to changes in the inotropic state during these phases. Indeed, $E_{E A 1}$ was positively correlated with $\mathrm{dP} / \mathrm{dt}_{\max }$ for all sheep with a median correlation coefficient $r$ of $0.78(\mathrm{Q} 1=0.68$; Q3 $=0.93)$, which is consistent with previous results on the peak-to-peak value of EA1 [2].

Table 2. Mean values of EA markers for all sheep during baseline and Valsalva phases (mean $\pm \mathrm{SD}, \mathrm{n}=18$ maneuvers). $* p<0.05$ vs baseline; $\dagger p<0.05$ vs CPP.

\begin{tabular}{|c|c|c|c|}
\hline & Baseline & CPP & Apnea \\
\hline$t_{E A 1}, \mathrm{~ms}$ & $30.2 \pm 14.3$ & $33.0 \pm 15.2^{*}$ & $28.6 \pm 9.5^{\dagger}$ \\
\hline$t_{E A 2}, \mathrm{~ms}$ & $285.3 \pm 22.1$ & $266.4 \pm 17.5^{*}$ & $284.6 \pm 15.1^{\dagger}$ \\
\hline$t_{E A 3 / 4}, \mathrm{~ms}$ & $-34.0 \pm 11.4$ & $-35.2 \pm 7.7$ & $-35.2 \pm 9.3$ \\
\hline$E_{E A 1}$ & $183.6 \pm 186.6$ & $168.4 \pm 200.1$ & $246.5 \pm 300.9^{* \dagger}$ \\
\hline$E_{E A 2}$ & $27.4 \pm 32.9$ & $20.9 \pm 20.4$ & $37.4 \pm 49.7^{\dagger}$ \\
\hline$E_{E A 3 / 4}$ & $0.4 \pm 0.5$ & $0.2 \pm 0.1^{*}$ & $0.9 \pm 1.2^{* \dagger}$ \\
\hline$w_{E A 1}, \mathrm{~ms}$ & $63.2 \pm 15.9$ & $65.2 \pm 11.3$ & $69.8 \pm 18.0^{*}$ \\
\hline$w_{E A 2}, \mathrm{~ms}$ & $41.6 \pm 8.5$ & $51.0 \pm 8.3^{*}$ & $41.8 \pm 12.5^{\dagger}$ \\
\hline
\end{tabular}



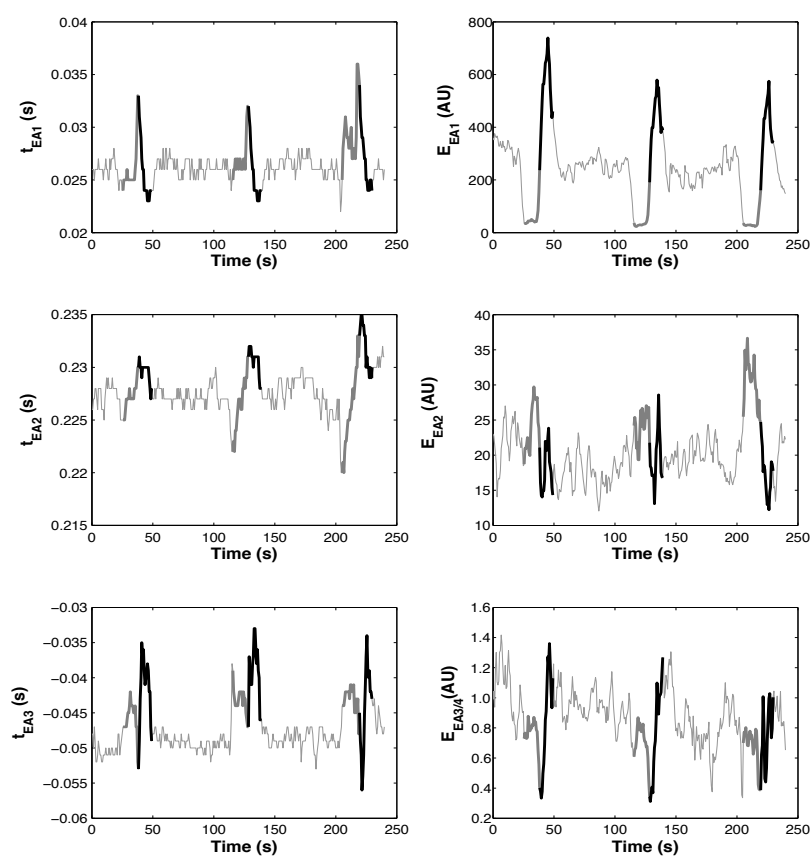

Figure 4. Evolution of EA indices during three consecutive Valsalva maneuvers in one sheep. Gray bold lines represent EA indices during the CPP step and black bold lines are EA indices during apnea. AU = arbitrary units.

\section{Conclusions}

This paper presented, to our knowledge, the first quantitative experimental analysis of intracardiac acceleration signals during acute cardio-respiratory variations. Significant modifications on the main EA markers were observed between the phases of Valsalva-like maneuvers, suggesting that these markers could be used for the characterization of respiratory events on patients that are implanted with an intracardiac accelerometer. Further evaluation is necessary to confirm these experimental results, on chronic, unanesthetized conditions.

\section{Acknowledgements}

This work was partially supported by BPI-FRANCE within the framework of the French Investments for the future program - INTENSE project. The authors would like to thank Dr Alain Bel for his constant availability and wise advice, as well as Martine Rancic, Julie Piquet and Adrien Lalot for their contributions on animal experimentation.

\section{References}

[1] Gill D, Gavrieli N, Intrator N. Detection and identification of heart sounds using homomorphic envelogram and self-organizing probabilistic model. In Proc. Computers in Cardiology. Sept. 25-28, 2005; 957-960.
[2] Plicchi G, Marcelli E, Parlapiano M, Bombardini T. Pea i and pea ii based implantable haemodynamic monitor: pre clinical studies in sheep. Europace 2002;4(1):49-54.

[3] Theres HP, Kaiser DR, Nelson SD, Glos M, Leuthold T, Baumann G, Sowelam S, Sheldon TJ, Stylos L. Detection of acute myocardial ischemia during percutaneous transluminal coronary angioplasty by endocardial acceleration. Pacing and clinical electrophysiology 2004;27(5):621-625.

[4] Donal E, Giorgis L, Cazeau S, Leclercq C, Senhadji L, Amblard A, Jauvert G, Burban M, Hernández A, Mabo P. Endocardial acceleration (sonr) vs. ultrasound-derived time intervals in recipients of cardiac resynchronization therapy systems. Europace Mar 2011;13(3):402-408.

[5] Giorgis L, Frogerais P, Amblard A, Donal E, Mabo P, Senhadji L, Hernández AI. Optimal algorithm switching for the estimation of systole period from cardiac microacceleration signals (sonr). IEEE Transactions on Biomedical Engineering 2012;59(11):3009-3015.

[6] Giorgis L, Frogerais P, Amblard A, Donal E, Mabo P, Senhadji L, Hernández AI. Optimal algorithm switching for the estimation of systole period from cardiac microacceleration signals (sonr). Biomedical Engineering IEEE Transactions on 2012;59(11):3009-3015.

[7] Hernandez A, Ziglio F, Amblard A, Senhadji L, Leclercq C, et al. Analysis of endocardial acceleration during intraoperative optimization of cardiac resynchronization therapy. In Engineering in Medicine and Biology Society (EMBC), 2013 35th Annual International Conference of the IEEE. IEEE, 2013; 7000-7003.

[8] Romero-Ugalde HM, Ojeda D, Rolle VL, Andreu D, Guiraud D, Bonnet JL, Henry C, Karam N, Hagege A, Mabo P, Carrault G, Hernandez AI. Model-based design and experimental validation of control modules for neuromodulation devices. Biomedical Engineering IEEE Transactions on 2015;ISSN 0018-9294.

[9] Gregory N, Audsley A, Lister D. Studies on the sympathetic nervous system: the valsalva like manoeuvre in sheep. Research in veterinary science 1981;30(3):284-287.

[10] Dumont J, Hernández A, Carrault G. Improving ecg beats delineation with an evolutionary optimization process. Biomedical Engineering IEEE Transactions on 2010; 57(3):607-615.

[11] Morimont P, Lambermont B, Desaive T, Janssen N, Chase G, D'Orio V. Arterial dp/dtmax accurately reflects left ventricular contractility during shock when adequate vascular filling is achieved. BMC cardiovascular disorders 2012; 12(1):13.

[12] Henning RJ, Levy MN. Effects of autonomic nerve stimulation, asynchrony, and load on dp/dtmax and on dp/dtmin. American Journal of Physiology Heart and Circulatory Physiology 1991;260(4):H1290-H1298.

Address for correspondence:

Alfredo I. Hernández

LTSI INSERM U1099. Université de Rennes 1.

Campus de Beaulieu. 35042. Rennes. France. 